\title{
Introducción a la función punto medio en continuos
}

\author{
María de Jesús López ${ }^{a}$, Patricia Pellicer-Covarrubias ${ }^{b *}$, \\ IVÁN SERAPIO RAMOS ${ }^{a}$ \\ ${ }^{a}$ Benemérita Universidad Autónoma de Puebla, Facultad de Ciencias \\ Físico-Matemáticas, Puebla, México. \\ ${ }^{b}$ Universidad Nacional Autónoma de México, Facultad de Ciencias, México, D.F. \\ México.
}

Resumen. El hiperespacio de arcos de un continuo fue definido por Sam B. Nadler, Jr. en 1978. Posteriormente, A. Soto estudió en 1999 el hiperespacio de arcos y singulares de un continuo, el cual en este artículo será denotado por $\mathcal{M}(X)$. En este trabajo introducimos la función punto medio y la función de puntos extremos en $\mathcal{M}(X)$, exponemos algunas de sus propiedades básicas, las comparamos y damos una caracterización de la continuidad de ambas funciones.

Palabras clave: función de puntos extremos; función de Whitney; función punto medio; hiperespacio de arcos y singulares; punto medio; puntos extremos.

MSC2010: 54B20, 54E40, 54F15.

\section{Introduction to the midpoint function in continua}

Abstract. The hyperspace of arcs of a continuum was defined by Sam B. Nadler, Jr. in 1978. Later, A. Soto studied in 1999 the hyperspace of arcs and singletons of a continuum, which we will denote in this paper by $\mathcal{M}(X)$. In this article we introduce a midpoint function and the end point function in $\mathcal{M}(X)$, we present some of their basic properties, we compare them and we give a characterization of the continuity of both of them.

Keywords: end point function; Whitney map; mid point function; hyperspace of arcs and singletons; midpoint, end point.

\footnotetext{
*E-mail: paty@ciencias.unam.mx

Recibido: 18 de enero de 2016, Aceptado: 11 de abril de 2016.

Para citar este artículo: M. de J. López, P. Pellicer-Covarrubias, I. Serapio Ramos, Introducción a la función punto medio en continuos, Rev. Integr. Temas Mat. 34 (2016), No. 1, 109-123.
} 


\section{Introducción}

Un continuo es un espacio métrico compacto, conexo y no vacío. Dado un continuo $X$ se pueden considerar familias de subconjuntos de $X$ con alguna propiedad en particular, a las que se les llama hiperespacios de $X$. Por ejemplo, para un continuo $X$ consideramos la colección

$$
2^{X}=\{A \subset X: A \text { es no vacío y cerrado en } X\} .
$$

También se considera la colección de todos sus subcontinuos $\left\{A \in 2^{X}: A\right.$ es conexo $\}$, la cual se denota por $C(X)$. Un hiperespacio más es $F_{1}(X)=\{\{x\}: x \in X\}$.

El estudio de los hiperespacios de continuos se ha concentrado principalmente en $2^{X}$ y $C(X)$; en [2] y [5] se puede consultar una amplia bibliografía al respecto. En [5, p. 601] Nadler sugiere que la investigación de otros hiperespacios podría ser relevante y, entre ellos, propone estudiar propiedades del hiperespacio de arcos de un continuo $X$ definido como $\mathcal{A}(X)=\left\{A \in 2^{X}: A\right.$ es un arco $\}$. Algunos años más tarde, Soto retoma en [6] la sugerencia de Nadler y define el hiperespacio de arcos y singulares, el cual se define como $\mathcal{M}(X)=\mathcal{A}(X) \cup F_{1}(X)$; ahí mismo Soto presenta varias propiedades de $\mathcal{M}(X)$ y desarrolla un material interesante, el cual lleva a A. Illanes a caracterizar la clase de las dendritas en términos del hiperespacio $\mathcal{M}(X)([1])$.

Por otro lado, una herramienta que ha resultado ser sumamente útil en el estudio de los hiperespacios son las funciones de Whitney ([8]). Con estas funciones en cierto sentido se "mide el tamaño" de los subcontinuos de un continuo y se demuestra una gran cantidad de resultados. En este escrito utilizamos las funciones de Whitney para introducir una función "natural" para el hiperespacio $\mathcal{M}(X)$ de un continuo $X$ : la función punto medio. Asimismo introducimos la función de puntos extremos en $\mathcal{M}(X)$ y la comparamos con la función punto medio. Adicionalmente presentamos algunas propiedades básicas de ambas funciones y demostramos que la continuidad de la función punto medio es equivalente a la continuidad de la función de puntos extremos.

\section{Definiciones y propiedades básicas}

Un subcontinuo de un continuo es un subespacio que también es un continuo. Un subespacio $A$ de un continuo es un arco si $A$ es homeomorfo al intervalo cerrado [0,1]. Dado un arco $A$ se dirá que un punto $p \in A$ es punto extremo de $A$ si existe un homeomorfismo $h:[0,1] \rightarrow A$ tal que $p=h(0)$. Definimos y denotamos la circunferencia por $S^{1}=\left\{(x, y) \in \mathbb{R}^{2}: x^{2}+y^{2}=1\right\} ;$ un subespacio de un continuo es una curva cerrada simple si es homeomorfo a la circunferencia. Sea $A$ un subconjunto de un espacio métrico; denotamos el diámetro de $A$ por diám $A$. Dado un subconjunto $A$ de los números reales, denotamos el máximo y el mínimo de $A$ por máx $A$ y mín $A$, respectivamente. Se dice que un espacio es no degenerado si contiene más de un punto.

Al hiperespacio $2^{X}$ que mencionamos en la Introducción se le dota de una topología, la cual se genera como sigue: consideremos una colección finita de subconjuntos $U_{1}, \ldots, U_{n}$ de $X$ y $n \in \mathbb{N}$, y denotamos:

$$
\left\langle U_{1}, \ldots, U_{n}\right\rangle=\left\{A \in 2^{X}: A \subset \bigcup_{i=1}^{n} U_{i} \text { y } A \cap U_{i} \neq \emptyset \text {, para cada } i \in\{1, \ldots, n\}\right\} .
$$


Ahora, pongamos:

$$
\mathcal{B}=\left\{\left\langle U_{1}, \ldots, U_{n}\right\rangle: U_{i} \text { es un abierto en } X, i \in\{1, \ldots, n\} \text { y } n \in \mathbb{N}\right\} .
$$

Se tiene que $\mathcal{B}$ es una base para una topología para $2^{X}$, la cual se conoce como topología de Vietoris. La demostración de este resultado se puede consultar en [4, Teorema 4.5]. El conjunto $2^{X}$ con la topología de Vietoris se llama el hiperespacio de cerrados de $X$.

Por otro lado, $C(X)$ considerado como subespacio de $2^{X}$ es conocido como el hiperespacio de subcontinuos de $X$. Se sabe que si $X$ es un continuo, entonces el hiperespacio $C(X)$ es conexo, compacto y metrizable, es decir, también es un continuo, [5, (1.13)]. Finalmente, $F_{1}(X)$ también se considera como subespacio de $2^{X}$ y lleva el nombre de hiperespacio de singulares de $X$.

Una caracterización útil de la convergencia en el hiperespacio $C(X)$ está dada en la siguiente proposición, cuya prueba se puede encontrar en [7, Lema 1.27, p. 16].

Proposición 2.1. Sean $X$ un continuo, $A \in C(X)$ y $\left\{A_{n}\right\}_{n \in \mathbb{N}}$ una sucesión de subcontinuos de $X$ que converge a $A$. Para cada $x \in X$ se tiene que $x \in A$ si y sólo si existe una sucesión $\left\{x_{n}\right\}_{n \in \mathbb{N}}$ tal que $x_{n} \rightarrow x$, para todo $n \in \mathbb{N}, x_{n} \in A_{n}$.

Dada una función continua entre continuos $f: X \rightarrow Y$, esta induce de manera natural una función entre los respectivos hiperespacios de subcontinuos, $C(f): C(X) \rightarrow C(Y)$, definida por $C(f)(A)=f(A)$, para cada $A \in C(X)$. Se sigue del Lema 13.3 de [2] que la función $C(f)$ es continua. Más aún, $C(f)$ es un homeomorfismo, un encaje o una función inclusión cuando $f$ es un homeomorfismo, un encaje o una función inclusión, respectivamente.

Como ya mencionamos, se denotará por $F_{1}(X)$ al conjunto de todos los elementos singulares del continuo $X$, es decir, $F_{1}(X)=\{\{x\}: x \in X\}$. De manera semejante se define $F_{2}(X)=\{\{x, y\}: x, y \in X\}$, el cual es nombrado el segundo producto simétrico de $X$ y es considerado como subespacio de $2^{X}$. Al igual que para los hiperespacios de subcontinuos, toda función continua entre continuos $f: X \rightarrow Y$ induce una función continua entre los segundos productos simétricos, la cual se denota por $F_{2}(f): F_{2}(X) \rightarrow F_{2}(Y)$ y está dada por $F_{2}(f)(A)=f(A)$, para cada $A \in F_{2}(X)$. Además, en caso de que $f$ sea un homeomorfismo, un encaje o una función inclusión, entonces $F_{2}(f)$ es un homeomorfismo, un encaje o una función inclusión, respectivamente. A continuación se enuncia una propiedad que simplifica el estudio de la convergencia en $F_{2}(X)$ ([6, Proposición 4.14, p. 40]).

Lema 2.2. Sean $X$ un continuo, $B \in F_{2}(X)$ y $\left\{B_{n}\right\}_{n \in \mathbb{N}}$ una sucesión en $F_{2}(X)$. Si $B=\{x, y\}$, entonces $B_{n} \rightarrow B$ si y sólo si existen sucesiones $\left\{x_{n}\right\}_{n \in \mathbb{N}} y\left\{y_{n}\right\}_{n \in \mathbb{N}}$ en $X$ tales que $x_{n} \rightarrow x, y_{n} \rightarrow y$, para cada $n \in \mathbb{N}, B_{n}=\left\{x_{n}, y_{n}\right\}$.

Recordemos que una herramienta muy conveniente para estudiar al hiperespacio $C(X)$ está dada por las funciones de Whitney. Uno de los resultados fundamentales en la teoría de hiperespacios garantiza la existencia de funciones de Whitney para el hiperespacio de subconjuntos cerrados no vacíos de un continuo. Este resultado se debe a Hassler 
Whitney, quien en 1933 fue el primero en construir este tipo especial de funciones en ciertos espacios de conjuntos [8]. Sin embargo, el primero en hacer uso de estas funciones, ahora llamadas funciones de Whitney, para el estudio de los hiperespacios fue J. L. Kelley en 1942 [3].

Dado un continuo $X$, una función de Whitney para el hiperespacio $C(X)$ es una función continua $\mu: C(X) \rightarrow \mathbb{R}$ tal que:

(a) para cada $x \in X, \mu(\{x\})=0 \mathrm{y}$,

(b) para cada $A, B \in C(X)$ con $A \subset B \neq A$, ocurre que $\mu(A)<\mu(B)$.

Observación 2.3. Sean $X$ y $Y$ continuos y $\mu$ una función de Whitney para el hiperespacio $C(X)$.

(1) La propiedad (b) en la definición de función de Whitney es equivalente a que $\mu$ sea una función estrictamente creciente, tomando el orden definido por la contención en $C(X)$ y el orden usual en $\mathbb{R}$.

(2) Dado $K \in C(X)$, se elige un punto $x \in K$. De la condición (a) en la definición de función de Whitney y del inciso (1) de esta observación, se tiene que $\mu(\{x\})=0 \leq$ $\mu(K) \leq \mu(X)$. Por lo tanto, es posible restringir el codominio de la función $\mu$ al intervalo $[0, \mu(X)]$ y obtener una función continua que sigue cumpliendo las propiedades (a) y (b) en la definición de función de Whitney. Así, en lo subsecuente se considerarán funciones de Whitney de la forma $\mu: C(X) \rightarrow[0, \mu(X)]$.

(3) Cuando $X$ es un continuo no degenerado se tiene que $\mu(X)>0$. Si se define otra función $\widetilde{\mu}$ como $\widetilde{\mu}(K)=\mu(K) / \mu(X)$, para cada $K \in C(X)$, entonces $\widetilde{\mu}: C(X) \rightarrow[0,1]$ es una función de Whitney con la propiedad de que $\widetilde{\mu}(X)=1$.

(4) Dada una inmersión $\varphi: Y \hookrightarrow X$ existe una única función continua $\widetilde{\mu}: C(Y) \rightarrow$ $[0, \mu(\varphi(Y))]$ tal que

$$
\mu \circ C(\varphi)=\jmath \circ \widetilde{\mu}
$$

donde $\jmath:[0, \mu(\varphi(Y))] \hookrightarrow[0, \mu(X)]$ es la respectiva función inclusión. Obsérvese que $\widetilde{\mu}: C(Y) \rightarrow[0, \mu(\varphi(Y))]$ también es una función de Whitney.

Lema 2.4. Las funciones mínimo y máximo, mín, máx: $2^{[0,1]} \rightarrow[0,1]$, son continuas.

Demostración. Note que para cada $F \in 2^{[0,1]}$ y cada $\alpha \in[0,1]$ tenemos que mín $F<\alpha$ si y sólo si existe $x \in F$ tal que $x<\alpha$. También se tiene que mín $F>\alpha$ si y sólo si $x>\alpha$, para cada $x \in F$. De esta forma,

$$
\operatorname{mín}^{-1}([0, \alpha))=\langle[0,1],[0, \alpha)\rangle \quad \text { y } \quad \min ^{-1}((\alpha, 1])=\langle(\alpha, 1]\rangle .
$$

Análogamente se verifica que

$$
\operatorname{máx}^{-1}([0, \alpha))=\langle[0, \alpha)\rangle \quad \text { y } \operatorname{máx}^{-1}((\alpha, 1])=\langle[0,1],(\alpha, 1]\rangle .
$$

Así, la imagen inversa de todo subbásico canónico de $[0,1]$ es un subconjunto abierto de $2^{[0,1]}$. De aquí, las funciones mínimo y máximo son continuas. 
Teorema 2.5. Cualquier continuo admite funciones de Whitney para su hiperespacio de continuos.

Demostración. Dado un continuo $(X, d)$, se elige un conjunto $\left\{x_{n}: n \in \mathbb{N}\right\}$ que sea denso en $X$. Para cada $n \in \mathbb{N}$, se considera la función continua $f_{n}: X \rightarrow[0,1]$ definida, para todo $x \in X$, como

$$
f_{n}(x)=\frac{1}{1+d\left(x, x_{n}\right)}
$$

Luego, para cada $n \in \mathbb{N}$, se define $\mu_{n}(A)=\operatorname{diám} f_{n}(A)=\operatorname{máx} f_{n}(A)-\operatorname{mín} f_{n}(A)$, para cada $A \in C(X)$. Por el Lema 2.4 la función $\mu_{n}: C(X) \rightarrow[0,1]$ es continua, para cada $n \in \mathbb{N}$. Aplicando el criterio $M$ de Weierstrass es posible definir una función continua $\mu: C(X) \rightarrow[0,1]$ como

$$
\mu(A)=\sum_{n=1}^{\infty} \frac{\mu_{n}(A)}{2^{n}} .
$$

Veamos que $\mu$ es una función de Whitney.

(a) Dado $x \in X$, tenemos que:

$$
\mu_{n}(\{x\})=\operatorname{diám} f_{n}(\{x\})=\operatorname{diám}\left\{f_{n}(x)\right\}=0,
$$

para cada $n \in \mathbb{N}$. Se sigue que $\mu(\{x\})=0$.

(b) Sean $A, B \in C(X)$ tales que $A \subset B \neq A$. Para todo $n \in \mathbb{N}$ ocurre que $f_{n}(A) \subset f_{n}(B)$ y, por lo tanto $\mu_{n}(A)=\operatorname{diám} f_{n}(A) \leq \operatorname{diám} f_{n}(B)=\mu_{n}(B)$. Así, para ver que $\mu(A)<$ $\mu(B)$ bastará demostrar que $\mu_{m}(A)<\mu_{m}(B)$, para algún $m \in \mathbb{N}$.

Consideremos un punto $y \in B \backslash A$ y $\varepsilon>0$ tales que $d(y, A)>\varepsilon$. Dado que el conjunto $\left\{x_{n}: n \in \mathbb{N}\right\}$ es denso en $X$, es posible hallar $m \in \mathbb{N}$ tal que $d\left(y, x_{m}\right)<\frac{\varepsilon}{2}$. De aquí,

$$
\frac{1}{1+\frac{\varepsilon}{2}}<\frac{1}{1+d\left(y, x_{m}\right)}=f_{m}(y) \text {. }
$$

Luego, dado $x \in A$ ocurre que $d\left(x, x_{m}\right) \geq d(x, y)-d\left(y, x_{m}\right)>\varepsilon-\frac{\varepsilon}{2}=\frac{\varepsilon}{2}$. En consecuencia,

$$
f_{m}(x)=\frac{1}{1+d\left(x, x_{m}\right)}<\frac{1}{1+\frac{\varepsilon}{2}}<f_{m}(y),
$$

lo cual implica que máx $f_{m}(A)<f_{m}(y) \leq$ máx $f_{m}(B)$. Como $A \subset B$, se tiene que mín $f_{m}(A) \geq \operatorname{mín} f_{m}(B)$. Así, diám $f_{m}(A)<\operatorname{diám} f_{m}(B)$, es decir, $\mu_{m}(A)<\mu_{m}(B)$. Con esto se concluye que $\mu(A)<\mu(B)$.

Por lo tanto, $\mu$ es una función de Whitney para $C(X)$.

Concluimos esta sección recordando otra herramienta muy útil de la teoría de los hiperespacios: los arcos ordenados. Dado un continuo $X$, un arco ordenado en $C(X)$ es una función continua $\alpha:[0,1] \rightarrow C(X)$ tal que, para cualesquiera $s, t \in[0,1]$ con $s<t$, se tiene que $\alpha(s) \subsetneq \alpha(t)$. Si $A, B \in C(X)$ son tales que $\alpha(0)=A$ y $\alpha(1)=B$, entonces diremos que $\alpha$ es un arco ordenado desde $A$ hasta $B$. La prueba de la existencia de los arcos ordenados en $C(X)$ está cuidadosamente presentada en los resultados 14.2 hasta 14.6 en [2]. Usaremos el siguiente resultado para demostrar el Teorema 4.12.

Vol. 34, No. 1, 2016] 
Teorema 2.6. Sea $X$ un continuo. Si $A, B \in C(X)$ son tales que $A \subset B \neq A$, entonces existe un arco ordenado en $C(X)$ desde $A$ hasta $B$.

\section{Hiperespacio de arcos y singulares}

Dado un continuo $X$ se definen los siguientes subespacios de $C(X)$ :

$$
\mathcal{A}(X)=\{A \in C(X): A \text { es un arco en } X\} \quad \text { y } \quad \mathcal{M}(X)=F_{1}(X) \cup \mathcal{A}(X) ;
$$

ellos se denominarán hiperespacio de arcos de $X$ e hiperespacio de arcos y puntos de $X$, respectivamente.

Lema 3.1. Si $X$ es un arco, entonces $\mathcal{M}(X)=C(X)$.

Demostración. Si $X$ es el intervalo [0,1], entonces el lema es claro, ya que todos sus subcontinuos son intervalos cerrados y acotados, es decir, son singulares o arcos en $[0,1]$. Ahora, dado que cualquier subcontinuo de $X$ es homeomorfo a algún subcontinuo de $[0,1]$, se sigue que los subcontinuos de $X$ son puntos o $\operatorname{arcos}$ en $X$. Por lo tanto, concluimos que $\mathcal{M}(X)=C(X)$.

Proposición 3.2. Sea $Y$ una curva cerrada simple, se tiene que:

$$
\mathcal{M}(Y)=C(Y) \backslash\{Y\} .
$$

Demostración. Claramente la proposición se satisface para $S^{1}$, pues cada uno de sus subcontinuos propios es homeomorfo a algún subcontinuo de la recta real y, por lo tanto, es un singular o un arco en $S^{1}$. Así, como todo subcontinuo propio de $Y$ es homeomorfo a un subcontinuo propio de $S^{1}$, se sigue que $\mathcal{M}(Y)=C(Y) \backslash\{Y\}$.

Proposición 3.3. Si $X$ y $Y$ son continuos y $\varphi: Y \hookrightarrow X$ es un encaje, entonces la función restricción $\left.C(\varphi)\right|_{\mathcal{M}(Y)}$, que denotaremos por $\mathcal{M}(\varphi)$, es un encaje de $\mathcal{M}(Y)$ en $\mathcal{M}(X)$. Además, si $\varphi$ es una función inclusión o un homeomorfismo, entonces $\mathcal{M}(\varphi)$ es una función inclusión o un homeomorfismo, respectivamente.

Demostración. Si $L$ es un singular o un arco en $Y$, entonces $\varphi(L)$ es un singular o un arco en $X$, es decir, si $L \in \mathcal{M}(Y)$ entonces $C(\varphi)(L) \in \mathcal{M}(X)$. Por lo tanto, $\mathcal{M}(\varphi)=$ $\left.C(\varphi)\right|_{\mathcal{M}(Y)}$ es la única función continua que hace que

$$
C(\varphi) \circ \widetilde{k}=k \circ \mathcal{M}(\varphi),
$$

donde $k$ y $\widetilde{k}$ son las respectivas funciones inclusión. Se observa que $\mathcal{M}(\varphi)$ es un encaje por ser restricción de $C(\varphi): C(Y) \hookrightarrow C(X)$, que es un encaje. Además, cuando $\varphi$ es una función inclusión se tiene que $C(\varphi)$ es una función inclusión y, consecuentemente, $\mathcal{M}(\varphi)$ también debe serlo. Finalmente, si $\varphi$ es un homeomorfismo, entonces, para cada $K \in \mathcal{M}(X)$ se tiene que $\varphi^{-1}(K) \in \mathcal{M}(Y)$ y $\mathcal{M}(\varphi)\left(\varphi^{-1}(K)\right)=\varphi\left(\varphi^{-1}(K)\right)=K$. Así, el encaje $\mathcal{M}(\varphi)$ es suprayectivo, y por lo tanto es un homeomorfismo.

[Revista Integración 
Proposición 3.4. Todo arco posee dos únicos puntos extremos.

Demostración. Sea $A$ un arco cualquiera, y consideremos un homeomorfismo $h:[0,1] \rightarrow$ $A$. Tenemos que $h(0)$ es un punto extremo de $A$. Por otra parte se considera el homeomorfismo $g:[0,1] \rightarrow A$ dado por $g(t)=h(1-t)$, para cada $t \in[0,1]$. Así, $g(0)=h(1)$ también es un punto extremo de $A$. Nótese que, para todo $t \in(0,1), A \backslash\{h(t)\}$ es un espacio disconexo, pues $A \backslash\{h(t)\}=h([0, t)) \cup h((t, 1])$, donde $h([0, t))$ y $h((t, 1])$ son conjuntos ajenos, no vacíos y abiertos en $A$. Por otro lado, si $p$ es un punto extremo de $A$, entonces $A \backslash\{p\}=h((0,1])$ es conexo. De aquí, $h(t)$ no puede ser un punto extremo de $A$ en el caso en que $t \in(0,1)$. Por lo tanto, $h(0)$ y $h(1)$ son los únicos puntos extremos del $\operatorname{arco} A$.

Observación 3.5. La Proposición 3.4 no sólo asegura la existencia de dos únicos puntos extremos en un arco $A$; de hecho, indica cómo hallarlos: dado un homeomorfismo $h$ : $[0,1] \rightarrow A, p=h(0)$ y $q=h(1)$ son los puntos extremos del arco $A$. Más aún, $p$ y $q$ son los únicos puntos de $A$ tales que $A \backslash\{p\}, A \backslash\{q\}$ y $A \backslash\{p, q\}$ son subespacios conexos.

Corolario 3.6. Sean $a, b \in \mathbb{R}$ con $a<b$. Si $A$ es un arco y $h:[a, b] \rightarrow A$ es un homeomorfismo, entonces $h(a)$ y $h(b)$ son los puntos extremos de $A$.

Definición 3.7. Dado un continuo $X$ definimos la función $\mathcal{E}: \mathcal{M}(X) \rightarrow F_{2}(X)$ como

$$
\mathcal{E}(K)= \begin{cases}K, & \text { si } K \in F_{1}(X), \\ \{p \in K: p \text { es un punto extremo de } K\}, & \text { si } K \in \mathcal{A}(X) .\end{cases}
$$

A esta función se la nombrará función de puntos extremos de $X$.

Observación 3.8. Para cada punto $p$ de un continuo $X$, se tiene que $p$ es el único "punto extremo" del singular $\{p\}$, esto es, $\mathcal{E}(\{p\})=\{p\}$.

Lema 3.9. Sean $A$ un arco, $p$ y $q$ sus puntos extremos y $K, L \in C(A)$. Se tiene:

(a) Si $p \in K$, entonces $p \in \mathcal{E}(K)$.

(b) Si $p \in K \cap L$, entonces $K \subset L$ o $L \subset K$.

(c) Si $p, q \in K$, entonces $K=A$.

(d) Para cualquier $x \in A \backslash\{p, q\}$ existen $A_{1}, A_{2} \in \mathcal{A}(A)$ tales que $A=A_{1} \cup A_{2}$ y $A_{1} \cap A_{2}=\{x\}$.

Demostración. Consideremos un homeomorfismo $h:[0,1] \rightarrow A$ tal que $h(0)=p$ y $h(1)=q$.

(a) Si $K$ es un singular, entonces es claro que $p \in \mathcal{E}(K)$. Ahora, supóngase que $K$ es un arco. Como $p \in K$, se sigue que $0 \in h^{-1}(K)$ y, en consecuencia, $h^{-1}(K)=[0, b]$ para 
algún $b \in[0,1]$. Así, como $\left.h\right|_{[0, b]}:[0, b] \rightarrow K$ es un homeomorfismo, se tiene que $p=h(0)$ es un punto extremo de $K$.

(b) Dado que $p \in K \cap L$, se tiene que $h^{-1}(K)=[0, b]$ y $h^{-1}(L)=[0, d]$, para algunos $b, d \in[0,1]$. Como $b \leq d$ o $d \leq b$, se sigue que $h^{-1}(K) \subset h^{-1}(L)$ ó $h^{-1}(L) \subset h^{-1}(K)$ y, por lo tanto, $K \subset L$ ó $L \subset K$.

(c) Si $p, q \in K$, entonces $0,1 \in h^{-1}(K)$. Así, $h^{-1}(K)=[0,1]$. Por lo tanto, $K=h([0,1])=$ A.

(d) Sea $t=h^{-1}(x)$. Como $x$ no es un punto extremo del arco $A$, se tiene que $t \in(0,1)$. Así, $[0, t]$ y $[t, 1]$ son intervalos no degenerados. Haciendo $A_{1}=h([0, t])$ y $A_{2}=h([t, 1])$ se obtiene que $A_{1}$ y $A_{2}$ son arcos y, además, $p$ y $x$ son los puntos extremos de $A_{1}$ y $x$ y $q$ son los puntos extremos de $A_{2}$.

Proposición 3.10. Sean $X$ y $Y$ continuos con funciones de puntos extremos $\mathcal{E}: \mathcal{M}(X) \rightarrow$ $F_{2}(X)$ y $\mathcal{E}^{\prime}: \mathcal{M}(Y) \rightarrow F_{2}(Y)$, respectivamente. Si $\varphi: Y \hookrightarrow X$ es un encaje, entonces

$$
\mathcal{E} \circ \mathcal{M}(\varphi)=F_{2}(\varphi) \circ \mathcal{E}^{\prime}
$$

Demostración. Si $L \in F_{1}(Y)$ entonces $\varphi(L) \in F_{1}(X)$ y, en consecuencia, $\mathcal{E}(\varphi(L))=$ $\varphi(L)=\varphi\left(\mathcal{E}^{\prime}(L)\right)$. Ahora, si $L \in \mathcal{A}(Y)$ entonces se puede elegir un homeomorfismo $h:[0,1] \rightarrow L$. Sean $p=h(0)$ y $q=h(1)$; se tiene que $\mathcal{E}^{\prime}(L)=\{p, q\}$. Si se define $h^{\prime}=\varphi \circ h$, entonces $h^{\prime}:[0,1] \rightarrow \varphi(L)$ es un homeomorfismo tal que $h^{\prime}(0)=\varphi(p)$ y $h^{\prime}(1)=\varphi(q)$ son los puntos extremos de $\varphi(L)$, esto es, $\mathcal{E}(\varphi(L))=\{\varphi(p), \varphi(q)\}=\varphi(\{p, q\})=\varphi\left(\mathcal{E}^{\prime}(L)\right)$. De aquí se concluye que $\mathcal{E} \circ \mathcal{M}(\varphi)=F_{2}(\varphi) \circ \mathcal{E}^{\prime}$.

Aplicando las Proposiciones 3.3 y 3.10 para el caso de funciones inclusión, se obtiene el siguiente resultado.

Corolario 3.11. Sean $X$ un continuo y $Y$ un subcontinuo cualquiera de $X$. Si $\mathcal{E}: \mathcal{M}(X) \rightarrow F_{2}(X)$ y $\mathcal{E}^{\prime}: \mathcal{M}(Y) \rightarrow F_{2}(Y)$ son las funciones de puntos extremos de $X$ y $Y$, respectivamente, entonces $\mathcal{E}^{\prime}=\left.\mathcal{E}\right|_{\mathcal{M}(Y)}$.

Definición 3.12. Un continuo $X$ tiene función de puntos extremos continua si la función $\mathcal{E}: \mathcal{M}(X) \rightarrow F_{2}(X)$ es continua.

Observación 3.13. Si $X$ es un continuo, entonces la función de puntos extremos $\mathcal{E}$ es continua en todo punto de $F_{1}(X)$.

Proposición 3.14. Tener función de puntos extremos continua es una propiedad topológica en los continuos.

[Revista Integración 
Demostración. Sea $\varphi: Y \rightarrow X$ un homeomorfismo entre continuos, y supóngase que $Y$ tiene función de puntos extremos continua. Como consecuencia de las Proposiciones 3.3 y 3.10 , se sigue que $\mathcal{M}(\varphi): \mathcal{M}(Y) \rightarrow \mathcal{M}(X)$ es un homeomorfismo tal que

$$
\mathcal{E} \circ \mathcal{M}(\varphi)=F_{2}(\varphi) \circ \mathcal{E}^{\prime}
$$

De esta manera, $\mathcal{E}=F_{2}(\varphi) \circ \mathcal{E}^{\prime} \circ \mathcal{M}(\varphi)^{-1}$. Por hipótesis, $\mathcal{E}^{\prime}$ es una función continua, de lo cual se sigue que $\mathcal{E}$ también es una función continua.

Teorema 3.15. Los arcos tienen función de puntos extremos continua.

Demostración. Debido a la Proposición 3.14, es suficiente demostrar que el intervalo [0,1] tiene función de puntos extremos continua. Esto se sigue del hecho de que, para cada $L \in C([0,1]), \mathcal{E}(L)=\{\operatorname{mín} L, \operatorname{máx} L\}$.

Teorema 3.16. Las curvas cerradas simples tienen función de puntos extremos continua.

Demostración. Sean $X$ una curva cerrada simple, $A \in \mathcal{M}(X)$ y una sucesión $\left\{A_{n}\right\}_{n \in \mathbb{N}}$ contenida en $\mathcal{M}(X)$ tal que $A_{n} \rightarrow A$. Considerando un punto $p \in X \backslash A$, se tiene que existe un conjunto abierto $U$ en $X$ tal que $A \subset U \subset c l_{X} U \subset X \backslash\{p\}$. Dado que $X$ es un continuo localmente conexo, se tiene que la componente de $U$ que contiene a $A$, la cual llamaremos $V$, también es un conjunto abierto. Nótese que $c l_{X} V \subset c l_{X} U \subset X \backslash\{p\}$. Pongamos $Y=c l_{X} V$. Se obtiene que $Y$ es un subcontinuo propio de $X$ y, por lo tanto, es un arco. Además, si $L \in \mathcal{M}(X)$ y $L \subset V$, entonces, por el Lema 3.1, $L \in C(Y)=\mathcal{M}(Y)$. De aquí que $A \in\langle V\rangle \cap \mathcal{M}(X) \subset \mathcal{M}(Y)$, es decir, $A$ es un punto interior de $\mathcal{M}(Y)$ en $\mathcal{M}(X)$. En consecuencia, existe $N \in \mathbb{N}$ tal que, si $n \in \mathbb{N}$ y $n \geq N$, entonces $A_{n} \in \mathcal{M}(Y)$. Por el Corolario 3.11 tenemos que $\left.\mathcal{E}\right|_{\mathcal{M}(Y)}$ es la función de puntos extremos de $Y$. Dado que $Y$ es un arco, dicha restricción debe ser continua. Así, como $\left\{A_{n+N}\right\}_{n \in \mathbb{N}}$ es una sucesión contenida en $\mathcal{M}(Y)$ que converge a $A \in \mathcal{M}(Y)$, se sigue que $\mathcal{E}\left(A_{n+N}\right) \rightarrow \mathcal{E}(A)$. Esto prueba que la función de puntos extremos $\mathcal{E}: \mathcal{M}(X) \rightarrow F_{2}(X)$ es continua.

\section{Funciones punto medio}

Definición 4.1. Sean $X$ un continuo y $\mu: C(X) \rightarrow[0, \mu(X)]$ una función de Whitney. Dados $L \in \mathcal{M}(X)$ y un punto $p \in X$ se dirá que $p$ es punto medio de $L$ respecto de $\mu$ si existen $L_{1}, L_{2} \in C(X)$ tales que $L=L_{1} \cup L_{2}, L_{1} \cap L_{2}=\{p\}$ y $\mu\left(L_{1}\right)=\mu\left(L_{2}\right)$.

Lema 4.2. Sean $X$ un continuo y $\mu$ una función de Whitney para $C(X)$. Si $L \in \mathcal{M}(X)$ y $p \in X$ es un punto medio de $L$ respecto de $\mu$, entonces $p \in L$. Más aún, si $L$ es un arco, entonces $p$ no es punto extremo de $L$.

Vol. 34, No. 1, 2016] 
Demostración. Es claro de la Definición 4.1 que si $p$ es punto medio de $L$ entonces $p \in L$. Supongamos ahora que $L$ es un arco, y que además $p$ es un punto extremo de $L$. Sean $L_{1}, L_{2} \in C(X)$ tales que $L=L_{1} \cup L_{2}, L_{1} \cap L_{2}=\{p\}$ y $\mu\left(L_{1}\right)=\mu\left(L_{2}\right)$. Nótese que $L_{1}$ y $L_{2}$ son subcontinuos de $L$ que contienen al punto extremo $p$. Del Lema 3.9 (b) se sigue que $L_{1} \subset L_{2}$ ó $L_{2} \subset L_{1}$. Dado que $\mu\left(L_{1}\right)=\mu\left(L_{2}\right)$, tenemos que $L_{1}=L_{2} \mathrm{y}$, en conscuencia, $L=L_{1} \cup L_{2}=L_{1} \cap L_{2}=\{p\}$, lo cual es una contradicción. Por lo tanto, $p$ no es punto extremo del arco $L$.

Lema 4.3. Sea $T=\{(x, y) \in[0,1] \times[0,1]: x \leq y\}$. Si $G: T \rightarrow C([0,1])$ es tal que $G(x, y)=[x, y]$, para cada $(x, y) \in T$, entonces $G$ es un homeomorfismo.

Demostración. Considérese la función $F: C([0,1]) \rightarrow T$, definida como $F(K)=$ (mín $K$, máx $K)$, para cada $K \in C([0,1])$. Debido al Lema 2.4, tenemos que $F$ es una función continua. Además, para todo $K \in C([0,1])$ ocurre que

$$
G(F(K))=G((\operatorname{mín} K, \text { máx } K))=[\operatorname{mín} K, \text { máx } K]=K,
$$

y para cada $(x, y) \in T$ se tiene que

$$
F(G((x, y)))=F([x, y])=(\operatorname{mín}[x, y], \operatorname{máx}[x, y])=(x, y) .
$$

Esto prueba que $F$ es una función biyectiva con función inversa $G$. Dado que $C([0,1])$ es un continuo y $T$ es un espacio métrico, se sigue que $F: C([0,1]) \rightarrow T$ es un homeomorfismo y, en consecuencia, $G: T \rightarrow C([0,1])$ también lo es.

Dados un continuo $X$ y $A \subset X$, se define el conjunto

$$
C(X, A)=\{B \in C(X): B \cap A \neq \emptyset\} .
$$

Lema 4.4. Sean $X$ un arco y $L \in C(X)$. Si para todo $K \in C(X, L)$ se define $f(K)=$ $K \cap L$, entonces $f: C(X, L) \rightarrow C(X)$ es una función continua.

Demostración. Considérese un homeomorfismo $h:[0,1] \rightarrow X$, y sean $c, d \in[0,1]$ tales que $h^{-1}(L)=[c, d]$. Nótese que, para cada $K \in C(X), K \cap L \neq \emptyset$ si y sólo si $h^{-1}(K) \cap[c, d] \neq \emptyset$. Por lo tanto, se puede definir la función $H: C(X, L) \rightarrow C([0,1],[c, d])$ como $H(K)=$ $h^{-1}(K)$. Nótese que $H$ es la restricción de $C\left(h^{-1}\right)$ a $C(X, L)$, así que $H$ es una función continua. Ahora, consideremos la función $f^{*}: C([0,1],[c, d]) \rightarrow C([0,1])$ definida, para cada $A \in C([0,1],[c, d])$, como $f^{*}(A)=A \cap[c, d]$. Nótese que

$$
f^{*}(A)=[\operatorname{máx}\{\operatorname{mín} A, c\}, \operatorname{mín}\{\operatorname{máx} A, d\}] .
$$

Como $A \cap[c, d] \neq \emptyset$, existe $x_{0} \in A \cap[c, d]$, es decir, mín $A \leq x_{0} \leq$ máx $A$ y $c \leq x_{0} \leq d$. Esto implica que máx $\{\operatorname{mín} A, c\} \leq x_{0} \leq \operatorname{mín}\{\operatorname{máx} A, d\}$. Usando la notación del Lema 4.3, $(\operatorname{máx}\{\operatorname{mín} A, c\}, \operatorname{mín}\{\operatorname{máx} A, d\}) \in T$ y $f^{*}(A)=G(\operatorname{máx}\{\operatorname{mín} A, c\}, \operatorname{mín}\{\operatorname{máx} A, d\})$. Esto prueba que la función $f^{*}$ está bien definida y es continua. Nótese que, para todo $K \in C(X, L)$,

$$
f(K)=K \cap L=h\left(h^{-1}(K) \cap[c, d]\right)=h\left(f^{*}(H(K))\right) .
$$

Se sigue que $f=C(h) \circ f^{*} \circ H$ y, por lo tanto, $f$ está bien definida y es continua. 
Proposición 4.5. Sean $A$ un arco y $p \in A$. Si $p$ no es un punto extremo de A, entonces existe una función de Whitney $\mu$ para $C(A)$ tal que $p$ es un punto medio de $A$ respecto de $\mu$.

Demostración. Como $p$ no es punto extremo de $A$, existen arcos $A_{1}$ y $A_{2}$ en $A$ tales que $A_{1} \cup A_{2}=A$ y $A_{1} \cap A_{2}=\{p\}$. Elíjanse funciones de Whitney $\mu_{1}: C\left(A_{1}\right) \rightarrow\left[0, \mu_{1}\left(A_{1}\right)\right]$ y $\mu_{2}: C\left(A_{2}\right) \rightarrow\left[0, \mu_{2}\left(A_{2}\right)\right]$. Por la Observación $2.3(3)$, se puede suponer que $\mu_{1}\left(A_{1}\right)=$ $\mu_{2}\left(A_{2}\right)=1$. Ahora, definimos la función $\mu: C(A) \rightarrow[0,2]$, para cada $K \in C(A)$, como

$$
\mu(K)= \begin{cases}\mu_{1}(K), & \text { si } K \subset A_{1}, \\ \mu_{2}(K), & \text { si } K \subset A_{2}, \\ \mu_{1}\left(K \cap A_{1}\right)+\mu_{2}\left(K \cap A_{2}\right), & \text { si } K \cap A_{1} \neq \emptyset \neq K \cap A_{2} .\end{cases}
$$

Nótese que $\mu$ se ha definido por partes en los conjuntos cerrados $C\left(A_{1}\right), C\left(A_{2}\right)$ y $C\left(A, A_{1}\right) \cap C\left(A, A_{2}\right)$. Probaremos que las definiciones coinciden en las intersecciones de dichos conjuntos.

Si $K \subset A_{1}$ y $K \subset A_{2}$, entonces $K \subset A_{1} \cap A_{2}=\{p\}$ y, por lo tanto, $K=\{p\}$. Esto implica que $\mu_{1}(K)=0=\mu_{2}(K)$.

Si $K \subset A_{1}$ y $K \cap A_{2} \neq \emptyset$, entonces $K \cap A_{2} \subset A_{1} \cap A_{2}=\{p\}$, y así $K \cap A_{2}=\{p\}$. En consecuencia, $\mu_{1}\left(K \cap A_{1}\right)+\mu_{2}\left(K \cap A_{2}\right)=\mu_{1}(K)+\mu_{2}(\{p\})=\mu_{1}(K)$. Análogamente se demuestra que si $K \subset A_{2}$ y $K \cap A_{1} \neq \emptyset$, entonces $\mu_{1}\left(K \cap A_{1}\right)+\mu_{2}\left(K \cap A_{2}\right)=\mu_{2}(K)$. Esto prueba que $\mu$ está bien definida. Además, $\mu$ se ha definido en $C\left(A_{1}\right), C\left(A_{2}\right)$ y $C\left(A, A_{1}\right) \cap C\left(A, A_{2}\right)$ como una función continua (véase Lema 4.4), así que $\mu$ es una función continua.

Ahora, veamos que $\mu$ es una función de Whitney.

(a) Si $x \in A$, entonces para algún $i \in\{1,2\}$ se tiene que $x \in A_{i}$. De aquí que $\mu(\{x\})=$ $\mu_{i}(\{x\})=0$.

(b) Sean $K, L \in C(A)$ tales que $K \subset L \neq K$. Si $L \subset A_{i}$, para algún $i \in\{1,2\}$, entonces $K \subset A_{i}$ y, por lo tanto,

$$
\mu(K)=\mu_{i}(K)<\mu_{i}(L)=\mu(L) .
$$

Por otro lado, considérese el caso en que $L \cap A_{1} \neq \emptyset \neq L \cap A_{2}$ y $K \subset A_{i}$ para algún $i \in\{1,2\}$. Podemos suponer que $L \cap A_{1}$ y $L \cap A_{2}$ son no degenerados, pues de lo contrario $L$ estaría contenido en $A_{1}$ o en $A_{2}$, y se remitiría al caso anterior. Como $L \cap A_{i} \in C\left(A_{i}\right)$ y $K \subset L \cap A_{i}$, se tiene que

$$
\mu(K)=\mu_{i}(K) \leq \mu_{i}\left(L \cap A_{i}\right)<\mu_{1}\left(L \cap A_{1}\right)+\mu_{2}\left(L \cap A_{2}\right)=\mu(L) .
$$

Por último, supóngase que $K \cap A_{1} \neq \emptyset \neq K \cap A_{2}$. Como $K \subset L \neq K$, entonces $L \cap A_{1} \neq \emptyset \neq L \cap A_{2}$ y, para algún $i \in\{1,2\}, K \cap A_{i} \subset L \cap A_{i} \neq K \cap A_{i}$. En consecuencia,

$$
\mu(K)=\mu_{1}\left(K \cap A_{1}\right)+\mu_{2}\left(K \cap A_{2}\right)<\mu_{1}\left(L \cap A_{1}\right)+\mu_{2}\left(L \cap A_{2}\right)=\mu(L) .
$$

Se deduce que $\mu(K)<\mu(L)$ siempre que $K \subset L \neq K$.

Vol. 34, No. 1, 2016] 
Teorema 4.6. Si $\mu$ es una función de Whitney para $C([0,1])$, entonces existe un único punto $t_{0} \in(0,1)$ que es punto medio de $[0,1]$ respecto de $\mu$. Mas aún, $t_{0}$ es el único punto de $[0,1]$ tal que $\mu\left(\left[0, t_{0}\right]\right)=\mu\left(\left[t_{0}, 1\right]\right)$.

Demostración. Si para cada $t \in[0,1]$ se define $f(t)=\mu([0, t])-\mu([t, 1])$, entonces la función $f:[0,1] \rightarrow \mathbb{R}$ es continua. Dados $s, t \in[0,1]$ con $s<t$, se cumple que $\mu([0, s])<$ $\mu([0, t])$ y $\mu([t, 1])<\mu([s, 1])$. De aquí, $f(s)<f(t)$. Esto prueba que $f$ es una función estrictamente creciente.

Nótese que $f(0)=-\mu([0,1])<0$ y $f(1)=\mu([0,1])>0$. Por el Teorema del Valor Intermedio existe un único punto $t_{0} \in(0,1)$ tal que $f\left(t_{0}\right)=0$, esto es, $\mu\left(\left[0, t_{0}\right]\right)=$ $\mu\left(\left[t_{0}, 1\right]\right)$. Pongamos $L_{1}=\left[0, t_{0}\right]$ y $L_{2}=\left[t_{0}, 1\right]$. Se tiene que $[0,1]=L_{1} \cup L_{2}, L_{1} \cap L_{2}=\left\{t_{0}\right\}$ y $\mu\left(L_{1}\right)=\mu\left(L_{2}\right)$. En consecuencia, $t_{0}$ es punto medio de $[0,1]$ respecto de $\mu$.

Ahora supóngase que $s_{0} \in[0,1]$ también es un punto medio de $[0,1]$ respecto de $\mu$. Elíjanse $M_{1}, M_{2} \in C([0,1])$ de manera que $[0,1]=M_{1} \cup M_{2}, M_{1} \cap M_{2}=\left\{s_{0}\right\}$ y $\mu\left(M_{1}\right)=$ $\mu\left(M_{2}\right)$. Nótese que $M_{1}$ y $M_{2}$ son conjuntos distintos entre sí, pues $M_{1} \cup M_{2} \neq M_{1} \cap M_{2}$. Dado que $\mu\left(M_{1}\right)=\mu\left(M_{2}\right)$, ninguno de estos subcontinuos puede ser igual a [0,1], esto es, tanto $M_{1}$ como $M_{2}$ son subcontinuos propios de [0,1]. Por el Lema 3.9.(c), ni $M_{1}$ ni $M_{2}$ pueden contener a ambos puntos extremos del intervalo [0,1]. Por lo tanto, se puede asumir, sin perder generalidad, que $0 \in M_{1} \backslash M_{2}$ y $1 \in M_{2} \backslash M_{1}$. De esta manera, si $a=$ mín $M_{2}$ y $b=$ máx $M_{1}$, entonces $M_{1}=[0, b]$ y $M_{2}=[a, 1]$. Luego, como $\left\{s_{0}\right\}=M_{1} \cap M_{2}=$ $[a, b]$, se tiene que $a=b=s_{0}$. Así, $f\left(s_{0}\right)=\mu\left(\left[0, s_{0}\right]\right)-\mu\left(\left[s_{0}, 1\right]\right)=\mu\left(M_{1}\right)-\mu\left(M_{2}\right)=0$, lo cual implica que $s_{0}=t_{0}$. Esto prueba la unicidad de $t_{0}$ como punto medio de $[0,1]$ respecto de $\mu$.

Notación. Dado un arco $A$, su conjunto de puntos extremos sera denotado por $E(A)$.

Teorema 4.7. Sean $X$ un continuo y $\mu$ una función de Whitney para $C(X)$. Para cada $L \in \mathcal{M}(X)$, existe un único punto $p \in L \backslash E(L)$ que es punto medio de $L$ respecto de $\mu$. Además, si $L$ es un arco con puntos extremos a y $b$, entonces $p$ es el único punto de $L$ para el cual existen $L_{1}, L_{2} \in \mathcal{A}(L)$ tales que $E\left(L_{1}\right)=\{a, p\}, E\left(L_{2}\right)=\{p, b\}$ y $\mu\left(L_{1}\right)=\mu\left(L_{2}\right)$.

Demostración. Claramente el teorema es cierto cuando $L \in F_{1}(X)$. Supóngase que $L$ es un arco en $X$ con puntos extremos $a$ y $b$. Consideremos un encaje $\varphi:[0,1] \hookrightarrow X$ tal que $\varphi([0,1])=L$. Más aún, por la Observación 3.5 se puede pedir que $\varphi(0)=a$ y $\varphi(1)=b$. Definiendo $\widetilde{\mu}(K)=\mu(\varphi(K))$, para cada $K \in C([0,1])$, se sigue de la Observación 2.3.(4) que $\tilde{\mu}$ es una función de Whitney para $C([0,1])$. Se sigue del Teorema 4.6 que existe un punto $t_{0} \in(0,1)$ tal que $t_{0}$ es el único punto medio de $[0,1]$ respecto de $\widetilde{\mu}$; además, $\widetilde{\mu}\left(\left[0, t_{0}\right]\right)=\widetilde{\mu}\left(\left[t_{0}, 1\right]\right)$. Ahora, pongamos $p=\varphi\left(t_{0}\right), L_{1}=\varphi\left(\left[0, t_{0}\right]\right)$ y $L_{2}=\varphi\left(\left[t_{0}, 1\right]\right)$. Se tiene que

$$
p \in L \backslash E(L), L_{1}, L_{2} \in \mathcal{A}(L), L=L_{1} \cup L_{2} \text { y } L_{1} \cap L_{2}=\{p\} .
$$

Nótese que $\mu\left(L_{1}\right)=\widetilde{\mu}\left(\left[0, t_{0}\right]\right)=\widetilde{\mu}\left(\left[t_{0}, 1\right]\right)=\mu\left(L_{2}\right)$. Esto implica que $p$ es punto medio de $L$ respecto de $\mu$. Además, $a=\varphi(0)$ y $p=\varphi\left(t_{0}\right)$ son los puntos extremos de $L_{1} \mathrm{y}$, por otro lado, $b=\varphi(1)$ y $p=\varphi\left(t_{0}\right)$ son los puntos extremos de $L_{2}$. 
Ahora supóngase que $q \in X$ también es punto medio de $L$ respecto de $\mu$, es decir, existen $K_{1}, K_{2} \in C(X)$ tales que $L=K_{1} \cup K_{2}, K_{1} \cap K_{2}=\{q\}$ y $\mu\left(K_{1}\right)=\mu\left(K_{2}\right)$. Definimos $s_{0}=\varphi^{-1}(q), M_{1}=\varphi^{-1}\left(K_{1}\right)$ y $M_{2}=\varphi^{-1}\left(K_{2}\right)$. Así, $M_{1}, M_{2} \in C([0,1]),[0,1]=M_{1} \cup M_{2}$, $M_{1} \cap M_{2}=\left\{s_{0}\right\}$ у $\widetilde{\mu}\left(M_{1}\right)=\mu\left(K_{1}\right)=\mu\left(K_{2}\right)=\widetilde{\mu}\left(M_{2}\right)$. De aquí, $s_{0}$ es un punto medio de $[0,1]$ respecto de $\widetilde{\mu}$. Debido a la unicidad de $t_{0}$ se sigue que $s_{0}=t_{0}$, y así $q=p$. Esto demuestra que $L$ tiene un único punto medio respecto de $\mu$.

Finalmente, supóngase que existen $q \in L$ y $K_{1}, K_{2} \in \mathcal{A}(L)$ tales que $E\left(K_{1}\right)=\{a, q\}$, $E\left(K_{2}\right)=\{q, b\}$ y $\mu\left(K_{1}\right)=\mu\left(K_{2}\right)$. Si $s_{0}=\varphi^{-1}(q)$, entonces $\varphi^{-1}\left(K_{1}\right)=\left[0, s_{0}\right], \varphi^{-1}\left(K_{2}\right)=$ $\left[s_{0}, 1\right]$. Así, $\widetilde{\mu}\left(\left[0, s_{0}\right]\right)=\mu\left(K_{1}\right)=\mu\left(K_{2}\right)=\widetilde{\mu}\left(\left[s_{0}, 1\right]\right)$. En consecuencia, al ser $t_{0}$ el único punto con esta propiedad se tiene que $t_{0}=s_{0} \mathrm{y}$, por lo tanto, $q=p$.

Debido a los Teoremas 2.5 y 4.7 se tiene la siguiente

Definición 4.8. Dados un continuo $X$ y una función de Whitney $\mu$ para $C(X)$, definimos la función $P_{\mu}: \mathcal{M}(X) \rightarrow X$ de la siguiente manera: para cada $L \in \mathcal{M}(X), P_{\mu}(L)$ es el único punto medio de $L$ respecto de $\mu$. A esta función se la nombrará función punto medio respecto de $\mu$.

Definición 4.9. Un continuo $X$ tiene funciones punto medio continuas si para cada función de Whitney $\mu$ para $C(X)$, la función $P_{\mu}: \mathcal{M}(X) \rightarrow X$ es continua.

Observación 4.10. Si $X$ es un continuo y $\mu$ es una función de Whitney para $C(X)$, entonces la función punto medio $P_{\mu}$ es continua en todo punto de $F_{1}(X)$.

Lema 4.11. Sean $X$ un continuo y $\mu$ una función de Whitney para $C(X)$. Si $F$ es un conjunto cerrado en $X$ y $U$ es un conjunto abierto en $X$ tales que $F \subset U$, entonces existe $\delta>0$ tal que, para cada subcontinuo $B$ de $X$ con $B \cap F \neq \emptyset$ y $\mu(B)<\delta$, se tiene que $B \subset U$.

Demostración. Sin pérdida de generalidad supondremos que $U \neq X$. Consideremos el conjunto $\mathcal{F}=\{B \in C(X): B \cap F \neq \emptyset$ y $B \backslash U \neq \emptyset\}$. Nótese que $2^{X} \backslash\langle X, F, X \backslash U\rangle=$ $\langle X \backslash F\rangle \cup\langle U\rangle$. De aquí, $\langle X, F, X \backslash U\rangle$ es un conjunto cerrado en $2^{X}$. Como $\mathcal{F}=\langle X, F, X \backslash$ $U\rangle \cap C(X)$, se sigue que $\mathcal{F}$ es compacto. Dado que $\mu$ es una función continua y $\mathcal{F} \subset C(X)$, existe $B_{0} \in \mathcal{F}$ tal que $\mu\left(B_{0}\right)=\operatorname{mín} \mu(\mathcal{F})$. Nótese que $B_{0} \cap F \neq \emptyset, B_{0} \backslash U \neq \emptyset$ y $F \subset U$, lo cual muestra que $B_{0}$ es no degenerado, y por lo tanto podemos definir $\delta=\mu\left(B_{0}\right)>0$. Si $B \in C(X)$ es tal que $B \cap F \neq \emptyset$ y $\mu(B)<\delta$, entonces $B \notin \mathcal{F}$. Se sigue que $B \backslash U=\emptyset$, esto es, $B \subset U$.

Concluimos este trabajo mostrando la relación que existe entre la función punto medio y la función de puntos extremos en continuos.

Teorema 4.12. Si $X$ es un continuo, entonces las siguientes proposiciones son equivalentes:

Vol. 34, No. 1, 2016] 
(1) X tiene funciones punto medio continuas;

(2) X tiene una función punto medio continua;

(3) X tiene función de puntos extremos continua.

Demostración. Es claro que (1) implica (2).

Veamos que (2) implica (3). Para esto supóngase que la función de puntos extremos $\mathcal{E}: \mathcal{M}(X) \rightarrow F_{2}(X)$ no es continua. Debido a la Observación 3.13, y puesto que $F_{2}(X)$ es un espacio métrico y compacto, podemos considerar $A \in \mathcal{A}(X)$ y una sucesión $\left\{A_{n}\right\}_{n \in \mathbb{N}}$ contenida en $\mathcal{A}(X)$ tales que $A_{n} \rightarrow A$ y $\mathcal{E}\left(A_{n}\right) \rightarrow D$, para algún $D \in F_{2}(X)$ con $D \neq \mathcal{E}(A)$. Esto implica que algún punto extremo de $A$ no pertenece a $D$. Sean $a$ y $b$ los puntos extremos de $A$ y supongamos que $a \notin D$. Como $A_{n} \rightarrow A$ y $\mathcal{E}\left(A_{n}\right) \rightarrow D$, se sigue de la Proposición 2.1 que existe una sucesión $\left\{a_{n}\right\}_{n \in \mathbb{N}}$ contenida en $X$ que converge al punto $a$, y para cada $n \in \mathbb{N}$ se tiene que $a_{n} \in A_{n} \backslash E\left(A_{n}\right)$. Ahora, sea $D=\{c, d\}$ con $c$ y $d$ posiblemente iguales. Dado que $\mathcal{E}\left(A_{n}\right) \rightarrow D$, por el Lema 2.2 existen sucesiones $\left\{c_{n}\right\}_{n \in \mathbb{N}}$ y $\left\{d_{n}\right\}_{n \in \mathbb{N}}$ en $X$ que convergen a $c$ y a $d$, respectivamente, de tal manera que $E\left(A_{n}\right)=\left\{c_{n}, d_{n}\right\}$, para todo $n \in \mathbb{N}$. Así, existen arcos contenidos en $A_{n}$,

$$
K_{n} \text { y } L_{n} \text {, tales que } A_{n}=K_{n} \cup L_{n} \text { y } K_{n} \cap L_{n}=\left\{a_{n}\right\} .
$$

Elíjanse conjuntos abiertos $U$ y $V$ en $X$ cuyas cerraduras sean ajenas y de manera que $a \in U$ y $D \subset V$. Como $a_{n} \rightarrow a, c_{n} \rightarrow c$ y $d_{n} \rightarrow d$, se puede hallar $N \in \mathbb{N}$ tal que, si $n \in \mathbb{N}$ y $n \geq N$, entonces $a_{n} \in U$ y $c_{n}, d_{n} \in V$. Notemos que el conjunto:

$$
F=\left\{a_{n}: n \in \mathbb{N}, n \geq N\right\} \cup\{a\}
$$

es cerrado y está contenido en el abierto $U$. Así, se puede aplicar el Lema 4.11 para cualquier función de Whitney $\mu: C(X) \rightarrow[0, \mu(X)]$ y obtener un número $\delta>0$ tal que, si $B \in C(X)$ con $B \cap F \neq \emptyset$ y $\mu(B)<\delta$, entonces $B \subset U$. Nótese que para cada $n \geq N$, $K_{n} \cap F \neq \emptyset \neq K_{n} \backslash U$, así que necesariamente ocurre que $\mu\left(K_{n}\right) \geq \delta$. Por el Teorema 2.6 podemos considerar un arco ordenado desde $\left\{a_{n}\right\}$ hasta $K_{n}$, luego por el Teorema del Valor Intermedio se puede hallar $K_{n}^{\prime} \in \mathcal{A}(X)$ tal que $a_{n} \in K_{n}^{\prime} \subset K_{n}$ y $\mu\left(K_{n}^{\prime}\right)=\delta$. Bajo un argumento similar se obtiene $L_{n}^{\prime} \in \mathcal{A}(X)$ tal que $a_{n} \in L_{n}^{\prime} \subset L_{n}$ y $\mu\left(L_{n}^{\prime}\right)=\delta$. Nótese que $K_{n}^{\prime} \cap L_{n}^{\prime}=\left\{a_{n}\right\}$, así que, si se define $M_{n}=K_{n}^{\prime} \cup L_{n}^{\prime}$ para cada $n \geq N$, entonces $M_{n} \subset A_{n}$ es un arco. Más aún, como $\mu\left(K_{n}^{\prime}\right)=\mu\left(L_{n}^{\prime}\right)$, se tiene que $P_{\mu}\left(M_{n}\right)=a_{n}$. Ahora se elige una función $\sigma: \mathbb{N} \rightarrow \mathbb{N}$ estrictamente creciente de manera que $K_{\sigma(n)}^{\prime} \rightarrow K$, $L_{\sigma(n)}^{\prime} \rightarrow L$ y $M_{\sigma(n)} \rightarrow M$, para algunos $K, L, M \in C(X)$. Obsérvese que $M=K \cup L$ y $M \subset A$. Además, $a \in K \cap L$, así que por el Lema 3.9.(b) $K \subset L$ ó $L \subset K$. Dado que $\mu(K)=\delta=\mu(L)$, se tiene que $K=L=M$. Por otro lado, $M$ es un arco, ya que $M \subset A$ y $\mu(M)=\delta>0$. Así, $M_{\sigma(n)} \rightarrow M$, pero $P_{\mu}\left(M_{\sigma(n)}\right)=a_{\sigma(n)} \rightarrow a \neq P_{\mu}(M)$, pues $a$ es un punto extremo de $M$. Consecuentemente, la función $P_{\mu}$ no es continua, esto para toda función de Whitney $\mu$.

Finalmente probaremos que (3) implica (1). Supóngase que la función de puntos extremos $\mathcal{E}: \mathcal{M}(X) \rightarrow F_{2}(X)$ es continua, y sea $\mu$ una función de Whitney para $C(X)$ arbitraria. Se demostrará que la función punto medio $P_{\mu}: \mathcal{M}(X) \rightarrow X$ es continua. Como consecuencia de la Observación 4.10, basta verificar que $P_{\mu}$ es continua en los puntos de $\mathcal{A}(X)$. 
Sean $A \in \mathcal{A}(X)$ y una sucesión $\left\{A_{n}\right\}_{n \in \mathbb{N}}$ contenida en $\mathcal{A}(X)$ tales que $A_{n} \rightarrow A$. Dado que $X$ es un continuo, podemos suponer que $P_{\mu}\left(A_{n}\right) \rightarrow p$, para algún punto $p \in X$. Resta probar que $p=P_{\mu}(A)$. Debido a la continuidad de la función de puntos extremos, se tiene que $\mathcal{E}\left(A_{n}\right) \rightarrow \mathcal{E}(A)$. Por lo tanto, si $\mathcal{E}(A)=\{a, b\}$ entonces, por el Lema 2.2, existen sucesiones $\left\{a_{n}\right\}_{n \in \mathbb{N}}$ y $\left\{b_{n}\right\}_{n \in \mathbb{N}}$ de manera que $a_{n} \rightarrow a, b_{n} \rightarrow b$ y, para todo $n \in \mathbb{N}$, $\mathcal{E}\left(A_{n}\right)=\left\{a_{n}, b_{n}\right\}$. Debido al Teorema 4.7 , para cada $n \in \mathbb{N}$ existen $K_{n}, L_{n} \in C\left(A_{n}\right)$ tales que $E\left(K_{n}\right)=\left\{a_{n}, P_{\mu}\left(A_{n}\right)\right\}, E\left(L_{n}\right)=\left\{P_{\mu}\left(A_{n}\right), b_{n}\right\}$ y $\mu\left(K_{n}\right)=\mu\left(L_{n}\right)$. Ahora, se elige una función estrictamente creciente $\sigma: \mathbb{N} \rightarrow \mathbb{N}$ de manera que $K_{\sigma(n)} \rightarrow K$ y $L_{\sigma(n)} \rightarrow L$ para algunos $K, L \in C(X)$. Nótese que $K, L \subset A$. Así, $K, L \in \mathcal{M}(X)$. Aplicando nuevamente la continuidad de la función de puntos extremos, se tiene que $\mathcal{E}\left(K_{\sigma(n)}\right) \rightarrow \mathcal{E}(K)$ y $\mathcal{E}\left(L_{\sigma(n)}\right) \rightarrow \mathcal{E}(L)$, esto es, $E(K)=\{a, p\}$ y $E(L)=\{p, b\}$. Además, $\mu(K)=\mu(L)$, pues para cada $n \in \mathbb{N}, \mu\left(K_{\sigma(n)}\right)=\mu\left(L_{\sigma(n)}\right)$. En consecuencia, $p=P_{\mu}(A)$. Esto prueba la continuidad de la función $P_{\mu}$.

Corolario 4.13. Sea $X$ un continuo. Si $\mu$ y $\gamma$ son funciones de Whitney para $C(X)$, entonces la función punto medio respecto de $\mu, P_{\mu}$, es continua si y sólo si la función punto medio respecto de $\gamma, P_{\gamma}$, es continua.

\section{Referencias}

[1] Illanes A., "Hyperspaces of arcs and two-point sets in dendroid", Topology Appl. 117 (2002), No. 3, 307-317.

[2] Illanes A. and Nadler S.B. Jr., Hyperspaces. Fundamentals and recent advances, Monographs and Textbooks in Pure and Applied Mathematics, 216, Marcel Dekker, Inc., New York, 1999.

[3] Kelley J. L., "Hyperspaces of a continuum", Trans. Amer. Math. Soc. 52 (1942), 22-36.

[4] Nadler S.B. Jr., Continuum theory. An introduction, Monographs and Textbooks in Pure and Applied Mathematics, 158, Marcel Dekker, Inc., New York, 1992.

[5] Nadler S.B. Jr., Hyperspaces of sets, Monographs and Textbooks in Pure and Applied Mathematics, 49, Marcel Dekker, Inc., New York-Basel, 1978.

[6] Soto A., "El hiperespacio de arcos de un continuo", Tesis de licenciatura, Universidad Nacional Autónoma de México, 1999, 92 p.

[7] Villanueva H., "Encajando conos en hiperespacios", Tesis de doctorado, Universidad Nacional Autónoma de México, 2012, 240 p.

[8] Whitney H., "Regular families of curves", Ann. of Math. (2) 34 (1933), No. 2, 244-270. 